\title{
Simulating the formation of galaxy clusters
}

\author{
Daisuke Nagai and Andrey V. Kravtsov
}

Department of Astronomy and Astrophysics, Center for Cosmological Physics, The University of Chicago, Chicago, IL 60637 USA email: daisuke,andrey@oddjob.uchicago.edu

\begin{abstract}
We study the effects of radiative cooling, star formation and stellar feedback on the properties and evolution of galaxy clusters using high-resolution Adaptive Mesh Refinement $\mathrm{N}$-body+gasdynamics simulations of clusters forming in the $\Lambda \mathrm{CDM}$ universe. Cooling leads to the condensation of gas in the inner regions of clusters, which in turn leads to steepening of the dark matter profile. The cooling gas is replaced by the higher-entropy gas from the outer regions, which raises the entropy and temperature of gas in the cluster core. The magnitude of these effects is likely overestimated in the current simulations because they suffer from the overcooling problem: a much larger fraction of baryons is in the form of cold gas and stars than is observed. We find that the thermal stellar feedback alone does not remedy this problem. Additional ad-hoc preheating can lower the amount of cold gas but a simple uniform preheating results in incorrect star formation history, as it delays the bulk of star formation until $z<1$. Our analysis shows that the overcooling in a cluster as a whole is really the overcooling in the central galaxy and its progenitors at high redshifts. This indicates that an additional heating mechanism that can continuously heat the gas in the cluster core is required to reproduce the observed cluster properties. Energy injection by the Active Galactic Nuclei, which may provide such heating, may thus be an important missing ingredient in the current theoretical models of cluster formation.
\end{abstract}

\section{Effects of cooling, star formation and feedback}

Below, we highlight some of the important effects of gas cooling and star formation on the properties of galaxy clusters:

- Gas cooling induces condensation of gas in the cluster core region $(\lesssim 100 \mathrm{kpc})$, and the baryon mass in the cluster core at $z=0$ is dominated by the stars associated with the central galaxy.

- The condensation of baryons causes contraction of dark matter and steepens the dark matter profile in the central region from $r^{-1.5}$ in the adiabatic runs to $r^{-2.0}$ in the simulations with cooling.

- Gas cooling induces inflow of high-entropy gas from the outside and raises the level of entropy and gas temperature in the cluster core region.

- Stellar feedback and/or additional preheating suppress the effects described above by regulating gas cooling. The magnitude of the effects on the distribution of dark matter, entropy and temperature of gas is, therefore, sensitive to the total fraction of baryons which cooled to form stars and cold gas clouds.

\section{The overcooling problem}

The cluster simulations with cooling and star formation revealed a significant problem: $35-55 \%$ of baryons within the virial radius are in the form of cold gas and stars at $z=0$, which is significantly larger than the observed value of $\lesssim 10-20 \%$ (e.g., Balogh 2001; Lin, Mohr \& Stanford 2003). This problem was previously reported in the simulations using 


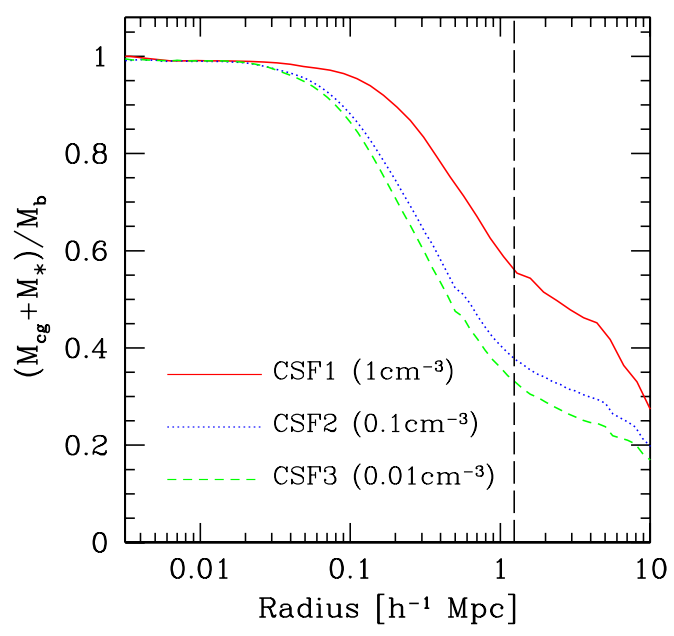

Figure 1. The cold gas + stellar mass fraction of the simulated cluster that includes cooling, star formation and stellar feedback. Three lines indicate the CSF runs with varying star formation: the star formation is allowed to proceed only in the region where the local gas density is larger than 1.0 (solid), 0.1 (dotted) and $0.01 \mathrm{~cm}^{-3}$ (dashed). The figure shows that $35-55 \%$ of baryons within the virial radius (indicated by the dashed line) are in the form of cold gas and stars at $z=0$, which is significantly larger than the observed value of $10-20 \%$.

the Smoothed Particle Hydrodynamics (SPH) simulations (e.g., Suginohara \& Ostriker 1998; Lewis et.al. 2000; Tornatore et.al. 2003). We find that the same problem exists in our higher-resolution Eulerian simulations that include metallicity-dependent radiative cooling. Moreover, we find that the cold and stellar mass fractions depend on the details of star formation implementaton. However, we were not able to solve the overcooling problem by varying parameters of thermal stellar feedback within reasonable limits, as shown in Figure 1.

\section{Effect of preheating}

Additional preheating can help resolve some of the discrepancies between cluster simulations and observations. Such preheating, presumably associated with the epoch of vigorous star formation, is often advocated as the solution to the overcooling problem and the discrepancies between the predicted and observed cluster scaling relations (e.g., Bialek et.al. 2001; Muanwong et.al. 2002, da Silva et.al. 2004). Our results, however, indicate that the additional preheating results in star formation history significantly different from observations, as shown in Figure 2. If the preheating occurs relatively early $(z \sim 3)$ the gas manages to cool back by $z \sim 1$, and the resulting star formation rate remains high $\left(\sim 1000 \mathrm{M}_{\odot} \mathrm{yr}^{-1}\right)$ at $z \lesssim 0.5$. This results in an incorrect star formation history: most of the cluster stars form in the last 7-10 billion years in stark contrast with observations, which indicate that less than $30 \%$ of cluster stars form during this epoch. If preheating occurs late, the amount of the required energy is unrealistic and the expected effect on the intergalactic medium is in contradiction to observations of the low-redshift Lyman- $\alpha$ forest. These results provide a strong argument against the simple uniform "preheating" scenario. 

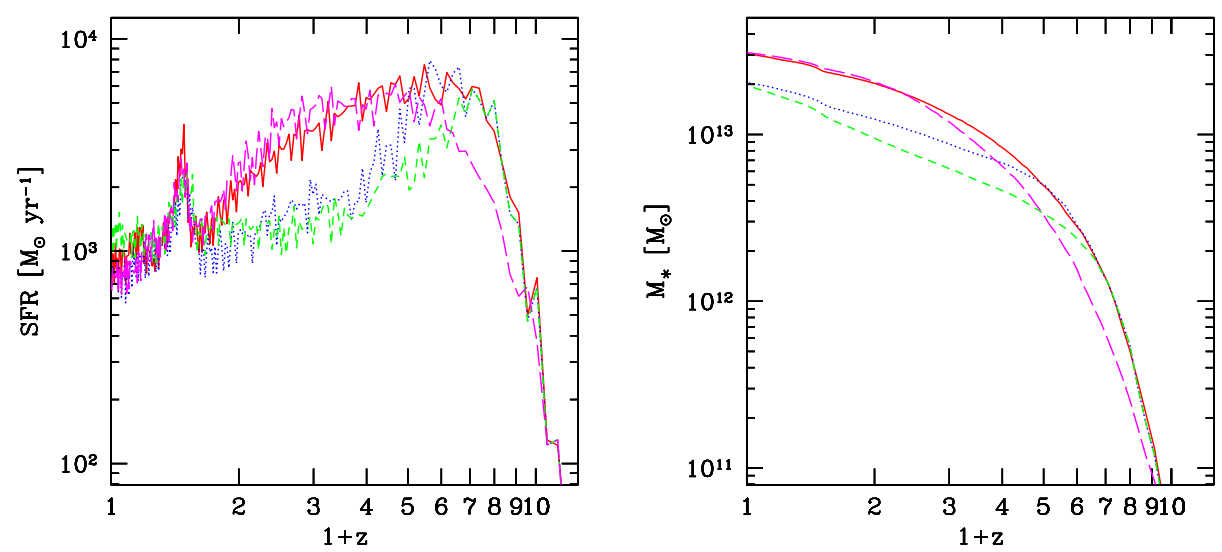

Figure 2. The star formation rate (left) and the stellar mass (right) as a function of redshift. These star formation history was constructed using stellar particles in the Lagrangian volume enclosed within the virial radius of the simulated cluster at $z=0$. Lines indicate the simulations that include cooling, star formation and stellar feedback (solid), and those with additional preheating of $0.3 \mathrm{keV} /$ particle at $z=4.5$ (dotted), $z=6$ (dashed) and $z=9$ (long-dashed).

\section{Discussions and conclusions}

The overcooling problem is a serious challenge for the cluster formation simulations. Our analysis shows that the overcooling in a cluster as a whole is really the overcooling in its core. In other words, the central cluster galaxies formed in simulations are too massive and bright compared to observations. Analysis of cluster evolution indicates that the problem sets in early at high redshifts, and is not limited to a single object or region. This indicates that an additional heating mechanism is necessary for reproducing the observed cluster properties. These results lend support to the idea that feedback from Active Galactic Nuclei is an important missing ingredient in the current simulations of cluster formation.

\section{References}

Balogh, M. L., Pearce, F. R., Bower, R. G., \& Kay, S. T. 2001, MNRAS, 326, 1228

Bialek, J. J., Evrard, A. E., \& Mohr, J. J. 2001, ApJ, 555, 597

da Silva, A. C., Kay, S. T., Liddle, A. R., \& Thomas, P. A. 2004, MNRAS, 348, 1401

Katz, N. \& White, S. D. M. 1993, ApJ, 412, 455

Lewis, G. F., Babul, A., Katz, N., Quinn, T., Hernquist, L., \& Weinberg, D. H. 2000, ApJ, 536, 623

Lin, Y. T., Mohr, J. J., \& Stanford, S. 2004, ApJ submitted (astro-ph/0402308)

Muanwong, O., Thomas, P. A., Kay, S. T., \& Pearce, F. R. 2002, MNRAS, 336, 527

Suginohara, T. \& Ostriker, J. P. 1998, ApJ, 507, 16

Tornatore, L., Borgani, S., Springel, V., Matteucci, F., Menci, N., \& Murante, G. 2003, MNRAS, 342,1025 